\title{
Transition from SPECT to PET myocardial perfusion imaging: A desirable change in nuclear cardiology to approach perfection
}

\author{
Vasken Dilsizian, $M^{a}$ \\ a Department of Diagnostic Radiology and Nuclear Medicine, University of Maryland School of \\ Medicine, Baltimore, MD
}

Received Feb 20, 2016; accepted Feb 20, 2016

doi: 10.1007/s12350-016-0475-6

\author{
"To improve is to change; to be perfect is to change \\ often." \\ Winston Churchill
}

In order to improve the current myocardial perfusion studies performed with SPECT, it is necessary to change, and that change may be achieved with PET instrumentation. Concentrated effort to break a new path with PET or to widen the one that already exists with SPECT is essential to remain innovative and current in medicine. PET represents significant technological advance over SPECT in terms of count rate, image resolution, and radiation exposure to patients. Advantages in the instrumentation, however, are only relevant if they significantly impact the diagnostic accuracy, patient prognosis, or the physician's confidence in the results. Accordingly, PET has to demonstrate a definite advantage over SPECT in order to play a significant role in the clinical care of patients.

Over the last four decades, radionuclide imaging has continuously expanded its role in noninvasive cardiac diagnostics. This progression can be attributed to parallel advances in SPECT and PET instrumentation, radiopharmaceuticals, and software development for quantitative analysis of myocardial perfusion, function, metabolism, and innervation. While both PET and SPECT provide valuable information regarding tomographic distribution of radiotracers, there are a number of physical differences that gives PET a clear competitive advantage over SPECT. Namely, PET has high

Reprint requests: Vasken Dilsizian, MD, Department of Diagnostic Radiology and Nuclear Medicine, University of Maryland Medical Center, 22 South Greene Street, Room N2W78, Baltimore, MD 21201-1595; vdilsizian@umm.edu

J Nucl Cardiol 2016;23:337-8.

$1071-3581 / \$ 34.00$

Copyright (C) 2016 American Society of Nuclear Cardiology. spatial and temporal resolution, short imaging protocols, reliable attenuation, and scatter correction; utilizes short-lived positron emitting radiotracers; and offers validated tracer kinetic models for quantifying absolute myocardial blood flow. ${ }^{1}$

Conventional SPECT approaches identify flowlimiting coronary artery narrowing by delineating the relative spatial distribution of myocardial blood flow during stress and at rest. A number of clinical trials have demonstrated the diagnostic and prognostic accuracy of gated myocardial perfusion SPECT, and more importantly, its relevance for patient management. While this approach has been clinically useful, it may underestimate balanced reduction in coronary artery blood flow, diffuse non-occlusive luminal coronary artery narrowing, or an occlusive lesion in the region with the highest relative radiotracer uptake by SPECT. Moreover, SPECT attenuation correction does not always rectify the underlying attenuation artifact. Sometimes, the SPECT attenuation algorithm itself may yield new artefactual defects that are related to reconstruction problems rather than true underlying myocardial physiology. PET permits reliable attenuation and scatter correction based on either external transmission sources or on CT images thereby enabling quantification of absolute myocardial blood flow in the heart.

While quantification of myocardial blood flow with PET has become an indispensable tool in cardiac research and a handful of clinical programs, SPECT continues to be the dominant technology used in clinical practice today. The main drawback for the PET technology is its cost. That is, not only the PET cameras are expensive, but also the production of radiotracers, which require either a cyclotron or an expensive generator for isotope production, are quite expensive to maintain, unless it is shared with other subspecialties, such as oncology. Recent introduction of F-18 labeled myocardial perfusion tracers may change the current paradigm 
of having an on-site cyclotron or an expensive generator to ordering unit doses of perfusion tracers on an "as needed" basis from regional medical cyclotrons. ${ }^{2}$ Moreover, the longer physical and biological half-life of $18 \mathrm{~F}$ would allow adequate image acquisition time to perform both treadmill and pharmacologic vasodilation stress studies, and a complete transition of nuclear laboratories from SPECT- to PET-based myocardial perfusion studies. As PET equipment becomes more widely utilized in cardiovascular disorders, it is expected that the additional clinical benefit of myocardial perfusion PET will be realized along with parallel escalation of novel positron-labeled radiotracers. ${ }^{3}$ There are already expanded applications of cardiac metabolism with FDG PET beyond myocardial viability, such as sarcoidosis, infection of prosthetic valves, and cardiac devices (pacemaker, AICD, and LVAD), and characterization of ventricular tachycardia substrate to guide ablation therapy. ${ }^{4,5}$

An important driver for cardiac PET comes from new directions in cardiovascular medicine that advocates a paradigm shift from detection and treatment of coronary artery disease to prediction and prevention. Current clinical application of myocardial perfusion SPECT is focused predominantly on the identification of isolated lesions, which can be treated by coronary revascularization. However, as aggressive medical therapy and/or prevention becomes effective alternative treatment options for coronary artery disease, the need arises to tailor the medication to the individual needs of patients. The unique capability of PET to quantify myocardial blood flow and metabolism makes it very attractive to study the influence of medical therapy in combination with common sense diet, lifestyle, and environmental factors on progression or regression of atherosclerosis and patient outcome. ${ }^{6}$ PET allows for a reliable quantitative comparison between a baseline study and follow-up studies, which is an important step for personalized medicine. ${ }^{7}$ However, in the absence of a multicenter study, similar to those published with FFR (e.g., FAME trials), that firmly establishes the incremental diagnostic and prognostic value of PET absolute hyperemic myocardial blood flow and flow reserve, it will be difficult for nuclear cardiology to take the lead as the noninvasive diagnostic study of choice to ultimately replace current FFR measurements in cardiac catheterization laboratories.

\section{References}

1. Schindler TH, Dilsizian V. PET-determined hyperemic myocardial blood flow: Further progress to clinical application. J Am Coll Cardiol. 2014;64(14):1476-8.

2. Dilsizian V, Taillefer R. Journey in evolution of nuclear cardiology: Will there be another quantum leap with the F-18 labeled myocardial perfusion tracers? J Am Coll Cardiol Img. 2012;5:1269-84.

3. Schindler TH, Dilsizian V. Cardiac positron emission tomography/computed tomography imaging of the renin-angiotensin system in humans holds promise for image-guided approach to heart failure therapy. J Am Coll Cardiol. 2012;60(24):2535-8.

4. Kim J, Feller ED, Chen W, Dilsizian V. FDG PET-CT imaging for LVAD associated infections. J Am Coll Cardiol CV Imaging. 2014;7(8):839-42.

5. Dickfeld $\mathrm{T}$, Lei $\mathrm{P}$, Dilsizian $\mathrm{V}$, et al Integration of threedimensional scar maps for ventricular tachycardia ablation with positron emission tomography-computed tomography. J Am Coll Cardiol Imaging. 2008;1:73-82.

6. Flachskampf FA, Dilsizian V. Leaning heavily on PET myocardial perfusion for prognosis. J Am Coll Cardiol Imaging. 2014;7(3):28891.

7. Dilsizian V, Narula J. Qualitative and quantitative scrutiny by regulatory process: Is the truth subjective or objective? J Am Coll Cardiol Imaging. 2009;2:1037-8. 